\title{
Article \\ Contrast Associated Acute Kidney Injury and Mortality in Older Adults with Acute Coronary Syndrome: A Pooled Analysis of the FRASER and HULK Studies
}

\author{
Rita Pavasini ${ }^{1}{ }^{*} \mathbb{D}$, Matteo Tebaldi ${ }^{1}$ (D) Giulia Bugani ${ }^{2}$, Elisabetta Tonet ${ }^{1}$, Roberta Campana ${ }^{1}$ (D), \\ Paolo Cimaglia ${ }^{3}$ (D), Elisa Maietti ${ }^{4,5}$, Giovanni Grazzi ${ }^{6}$, Graziella Pompei ${ }^{1}$ (D) , Gioele Fabbri ${ }^{1}$, Alessio Fiorio ${ }^{1}{ }^{(D)}$, \\ Andrea Rubboli ${ }^{7}$, Gianni Mazzoni ${ }^{8}$, Francesco Vitali ${ }^{1}$, Matteo Serenelli ${ }^{1}$ (D), Gianluca Campo 1,3 \\ and Simone Biscaglia ${ }^{1}$
}

Citation: Pavasini, R.; Tebaldi, M.; Bugani, G.; Tonet, E.; Campana, R.; Cimaglia, P.; Maietti, E.; Grazzi, G.; Pompei, G.; Fabbri, G.; et al. Contrast Associated Acute Kidney Injury and Mortality in Older Adults with Acute Coronary Syndrome: A Pooled Analysis of the FRASER and HULK Studies. J. Clin. Med. 2021, 10, 2151. https://doi.org/10.3390/jcm10102151

Academic Editors: Nicola Cosentino and Salvatore De Rosa

Received: 29 March 2021

Accepted: 27 April 2021

Published: 16 May 2021

Publisher's Note: MDPI stays neutral with regard to jurisdictional claims in published maps and institutional affiliations.

Copyright: (c) 2021 by the authors Licensee MDPI, Basel, Switzerland. This article is an open access article distributed under the terms and conditions of the Creative Commons Attribution (CC BY) license (https:/ / creativecommons.org/licenses/by/ $4.0 /)$.
1 Cardiovascular Institute, Azienda Ospedaliero-Universitaria di Ferrara, 44124 Ferrara, Italy; tblmtt@unife.it (M.T.); tonet.elisabetta@gmail.com (E.T.); robertacampana22@gmail.com (R.C.); graziella.pompei@outlook.it (G.P.); gioele.fabbri2@gmail.com (G.F.); alessiofiorio@virgilio.it (A.F.); Francesco.vitali90@gmail.com (F.V.); matteoserenelli@gmail.com (M.S.); cmpglc@unife.it (G.C.); simone.biscaglia@gmail.com (S.B.)

2 U.O.C. Cardiologia, Ospedale Maggiore, Largo Nigrisoli 2, 40133 Bologna, Italy; giulia.bugani@gmail.com 3 Maria Cecilia Hospital, GVM Care \& Research, 48033 Cotignola, Italy; paolocimaglia88@gmail.com

4 Department of Medical Science, University of Ferrara, 44121 Ferrara, Italy; e.maietti@ospfe.it

5 Department of Biomedical and Neuromotor Sciences, University of Bologna, 40127 Bologna, Italy

6 Center of Sport and Exercise Sciences, University of Ferrara, 44121 Ferrara, Italy; giovanni.grazzi@unife.it

7 Division of Cardiology, S. Maria Delle Croci Hospital, 48121 Ravenna, Italy; andrea.rubboli@auslromagna.it

8 Public Health Department, Azienda USL di Ferrara, 44121 Ferrara, Italy; mzo@unife.it

* Correspondence: pvsrti@unife.it

\begin{abstract}
Whether contrast-associated acute kidney injury (CA-AKI) is only a bystander or a risk factor for mortality in older patients undergoing percutaneous coronary intervention (PCI) is not well understood. Data from FRASER (NCT02386124) and HULK (NCT03021044) studies have been analysed. All patients enrolled underwent coronary angiography. The occurrence of CA-AKI was defined based on KDIGO criteria. The primary outcome of the study was to test the relation between CA-AKI and 3-month mortality. Overall, 870 older ACS adults were included in the analysis (mean age $78 \pm 5$ years; $28 \%$ females). CA-AKI occurred in 136 (16\%) patients. At 3 months, 13 (9.6\%) patients with CA-AKI died as compared with $13(1.8 \%)$ without it $(p<0.001)$. At multivariable analysis, CA-AKI emerged as independent predictor of 3-month mortality (HR 3.51, 95\%CI 1.05-7.01). After 3 months, renal function returned to the baseline value in $78(63 \%)$ with CA-AKI. Those without recovered renal function $(n=45,37 \%)$ showed an increased risk of mortality as compared to recovered renal function and no CA-AKI subgroups (HR 2.01, 95\%CI 1.55-2.59, $p=0.009$ and HR 2.71, 95\%CI $1.45-5.89, p<0.001$, respectively). In conclusion, CA-AKI occurs in a not negligible portion of older MI patients undergoing invasive strategy and it is associated with short-term mortality.
\end{abstract}

Keywords: acute coronary syndrome; percutaneous coronary intervention; contrast induced acute kidney injury; older patients; mortality; physical performance

\section{Introduction}

Several studies documented a strong association between contrast-associated acute kidney injury (CA-AKI) and an increased risk of adverse events, including mortality, need for dialysis, and hospital readmission for heart failure [1-6]. At the same time, both advanced age and hospital admission for myocardial infarction (MI) are significant risk factors for the occurrence of CA-AKI $[7,8]$. Lower baseline creatinine clearance, lower ejection fraction, high prevalence of heart failure at admission, and more complex coronary anatomy disease with severe calcifications are all common characteristics of older MI 
patients and all these factors further increase the risk of CA-AKI [9]. Thus, it is not surprising that nearly one out of five older adults undergoing cardiac catheterization for acute MI experience CA-AKI with associated increased short-term mortality [10]. However, recent evidence challenged the dogma that considers CA-AKI to be a direct cause of serious adverse events as mortality, need of dialysis, or persistent kidney impairment, suggesting that it could be only a marker (or a more frequent complication) of patients at higher risk for these complications [2,11-13]. Trying to better elucidate the relationship between age, CA-AKI and mortality, we analyzed the occurrence and prognostic implication of CA-AKI in two prospective studies focused on patients aged 70 years or more admitted to hospital for acute coronary syndrome (ACS) and undergoing invasive treatment.

\section{Materials and Methods}

\subsection{Study Design}

The present analysis was carried out by using the data of the populations of two different studies. The first one was the "Frailty in Elderly Patients Receiving Cardiac Interventional Procedures" (FRASER) study (NCT02386124) [14-16]. In brief, this multicentre, observational, prospective study analysed the frailty status of 402 adults aged $\geq 70$ years admitted to four Italian hospitals, with a diagnosis of ACS [14-16]. The second one was the "Physical Activity Intervention for Patients with Reduced Physical Performance After Acute Coronary Syndrome" (HULK) study (NCT03021044) [17-19]. In the latter randomized clinical trial, 485 adults aged $\geq 70$ years admitted to three Italian hospitals for ACS were screened for physical performance at hospital discharge and then, if fulfilling inclusion and exclusion criteria, were randomized to health education or exercise intervention [17-19].

\subsection{Study Measurements}

A large amount of clinical and management data, including demographics, previous medical history, comorbidities, echocardiographic and laboratory data, and treatments were collected in both studies. Starting from the individual dataset of both studies, a single database was generated under the supervision of the principal investigator (GC). Mehran risk score was not a prespecified variable available in the database. It is ad hoc calculated for the present analysis based on clinical and procedural variables available in the database [20]. The variables for Mehran risk score computation were age greater than 75 years, hypotension, congestive heart failure, intra-aortic balloon pump, serum creatinine, diabetes, anemia, and volume of contrast [20]. Left ventricular ejection fraction was calculated by the modified biplane Simpson method.

\subsection{Procedures for $C A-A K I$ Prevention}

Indication for coronary artery angiography and percutaneous coronary intervention (PCI) were in keeping with international guidelines and institutional protocols $[15,17]$. Angiographies and PCI were performed by experienced operators in high-volume hub centers. The operators and centers shared protocols to minimize contrast media administration and the risk of CA-AKI. All patients received pre- and post-hydration. ST segment elevation myocardial infarction (STEMI) patients were treated with a saline infusion at a rate of 0.5 to $1 \mathrm{~mL} / \mathrm{kg}$ per hour from admission and up to $24 \mathrm{~h}$ after the procedure. Saline infusion at a rate of 0.5 to $1 \mathrm{~mL} / \mathrm{kg}$ per hour for $12 \mathrm{~h}$ before and up to $24 \mathrm{~h}$ after the procedure was administered to NSTEACS patients, or in the case of staged procedure. Automatic systems for contrast injection and low-osmolal contrast agent (Omnipaque 350, GE Healthcare, Chicago, IL, USA) were used in all centers. The general recommendation was to estimate the maximal allowable contrast dose (MACD), defined as: $5 \mathrm{~mL} \times$ body weight (in $\mathrm{kg}$ )/ serum creatinine (in $\mathrm{mg} / \mathrm{dL}$ ), with a maximum dose of $300 \mathrm{~mL}$ [21] and not to exceed it. If necessary, staged procedures (after at least 3 days) were suggested. 


\subsection{Definition of $C A-A K I$}

The occurrence of CA-AKI was prospectively assessed. Serum creatinine (Beckman Coulter Inc., Brea, CA, USA) was measured at hospital admission (baseline value before any procedure) and every day until hospital discharge. Serum creatinine was expressed as $\mathrm{mg} / \mathrm{dl}$ and creatine clearance was estimated by the Cockroft-Gault formula. CA-AKI was defined based on the Kidney Disease Improving Global Outcomes (KDIGO) criteria: increase in peak serum creatinine of $\geq 0.3 \mathrm{mg} / \mathrm{dL}$ from baseline or $\geq 1.5$ times baseline within $48-72 \mathrm{~h}$ from the invasive procedure [22]. In patients with CA-AKI, serum creatine was measured again after 3 months [22]. If the 3-month value returned to baseline value (according KDIGO criteria) the patient was classified as recovered renal function. On the contrary, if the values persisted in being altered (according KDIGO criteria), the patients were classified as not having recovered renal function [22]. An independent central core-lab reviewed and adjudicated the occurrence of CA-AKI using original source documents.

\subsection{Clinical Follow-Up}

All patients received follow-up visits at 1, 6, 12 months, and then yearly. Patients with CA-AKI received an additional 3-month visit with blood sample withdrawn for serum creatinine determination. Patients were examined and asked about the occurrence of adverse events $[15,17]$. Source documentation regarding each adverse event was collected $[15,17]$. The clinical events committee, whose members were unaware of the patients characteristics, centrally evaluated all events. Clinical follow-up was censored in April 2020. The FRASER study enrolled patients from December 2014 to October 2016, then a 3 -year follow-up was available for all (median 1540 (1310-1700) days). On the contrary, the HULK study included patients from January 2017 to April 2018, then a 2-year follow-up was available for all (median 840 (705-1015) days). Overall, the median follow-up of the study population of the present analysis was 1023 (740-1446) days.

\subsection{Outcomes}

The primary outcome of the present analysis was all-cause mortality. The first aim of the study was to test the relationship between CA-AKI and 3-month mortality. The second aim was to assess the relationship between recovered and not recovered renal function and all-cause mortality beyond the 3-month time point. The major predictors of CA-AKI were identified.

\subsection{Statistical Analysis}

Continuous data were tested for normal distribution with the Kolmogorov-Smirnov test. Normally distributed values were presented as mean \pm standard deviation (SD) and compared using $t$-test, otherwise median value (interquartile range, IQR) and the Mann-Whitney $U$ test were used. Categorical variables were summarized in terms of counts and percentages and were compared by using the Fisher's exact test. The estimation of the cumulative primary endpoint rate was found by using the Kaplan-Meier method, and groups were compared with the log-rank test. To better discriminate the effect of CA-AKI on short vs. long-term mortality, we performed analyses with the landmark set at 3 months. The 3-month time point was selected based on previous studies [23]. All variables included in Table 1 were tested using univariate Cox regression as predictors of adverse events. Variables showing a $p$-value $<0.05$ were included in a multivariable model in association with CA-AKI with backward stepwise modelling approach. Variables remaining significant with a threshold $p$-value $\leq 0.05$ were retained as final predictors. Cox regression analyses were performed for 3-month mortality and for mortality beyond the 3-month time point. The independent risk factors for CA-AKI were calculated using logistic regression. All tests were 2 -sided, and the statistical significance was defined as $p<0.05$. All analyses were performed with Stata 13 by the staff of the Center for Clinical Epidemiology of the School of Medicine at the University of Ferrara (Ferrara, Italy). 
Table 1. Baseline characteristics.

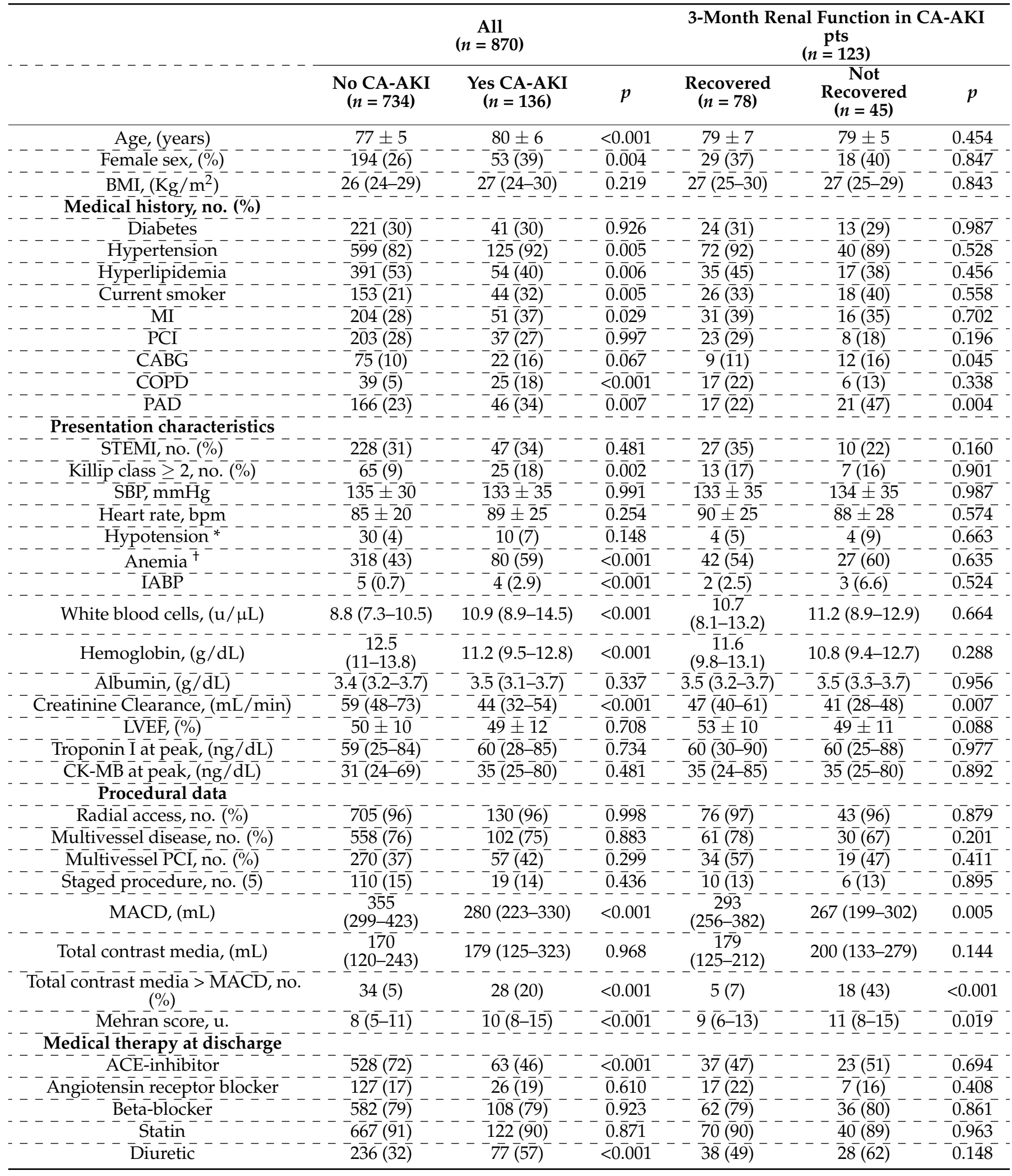

CA-AKI: contrast associated acute kidney injury. BMI: body mass index. MI: myocardial infarction. PCI: percutaneous coronary intervention. CABG: coronary artery bypass graft. COPD: chronic obstructive pulmonary disease. PAD: peripheral artery disease. STEMI: ST-segment elevation myocardial infarction. SBP: systolic blood pressure. IABP: intra-aortic balloon pump. LVEF: left ventricular ejection fraction. MACD: maximum allowed contrast dose. ACE: angiotensin converting enzyme. *: hypotension is defined as a systolic blood pressure $\leq 90 \mathrm{mmHg}$. t: anemia was defined as hemoglobin $<13 \mathrm{~g} / \mathrm{dL}$ for men and $<12 \mathrm{~g} / \mathrm{dL}$ for women. 


\section{Results}

Starting from 887 older ACS patients, we excluded $5(0.6 \%)$ patients on chronic dialysis, $3(0.3 \%)$ patients not undergoing PCI and $9(1 \%)$ patients due to missing data. Thus, the final study population involved $870(98 \%)$ older ACS adults undergoing PCI (Table 1). The mean age was $78 \pm 5$ years and 247 (28\%) patients were females. Overall, 275 (32\%) patients were admitted for STEMI. According to KDIGO definition, CA-AKI was observed in $136(16 \%)$ patients. It was severe (stage 3 of AKI, defined as 3-time baseline creatinine, or serum creatinine $>4 \mathrm{mg} / \mathrm{dL}$ or initial of dialysis [22]) in 6 patients and moderate in 17 patients (stage 2 of AKI, defined as 2 to 2.9-time baseline creatinine [22]). Of note, three of the patients with severe AKI $(2.2 \%)$ required dialysis during index hospitalization. Several baseline characteristics differed between patients with or without CA-AKI (Table 1). Patients with CA-AKI required a longer hospitalization stay (8 (7-10) days vs. 5 (4-6) days, $p<0.001)$.

\subsection{CA-AKI and 3-Month Mortality}

At 3 months, all-cause mortality occurred in $26(3 \%)$ patients. Thirteen $(9.6 \%)$ patients with CA-AKI died as compared with $13(1.8 \%)$ of those without $(p<0.001)$. The unadjusted cumulative occurrence of 3-month mortality was significantly different between subgroups, being higher in those with CA-AKI $(p<0.001)$ (Figure 1). At the univariate analysis, several clinical, laboratory, and angiographic variables, as well as CA-AKI, were associated with 3-month mortality (Table 2). At multivariable analysis, after correction for potential confounding factors, CA-AKI emerged as an independent predictor of 3-month death (HR 3.51, 95\%CI 1.05-7.01) (Table 2).

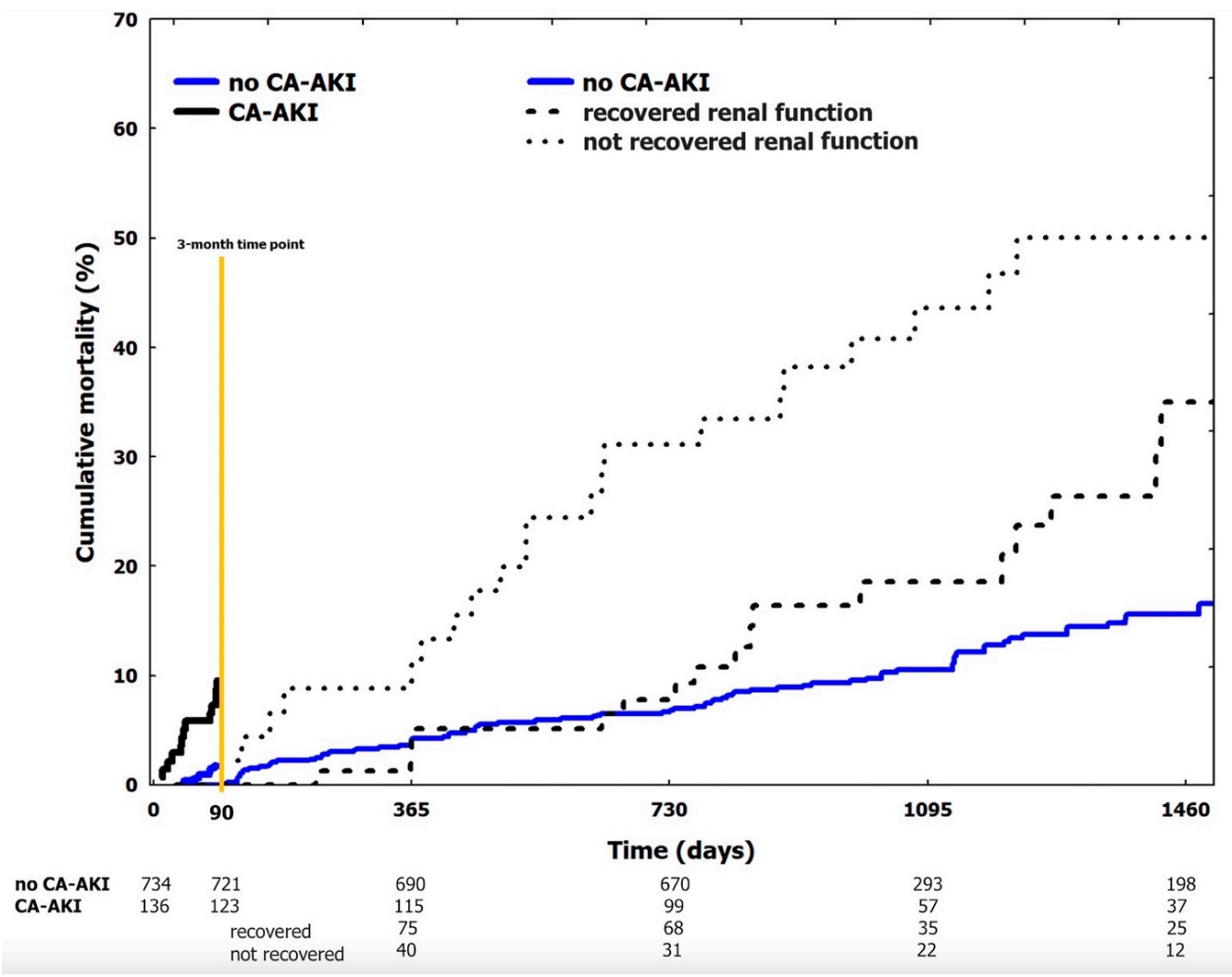

Figure 1. Cumulative occurrence of mortality (landmark analysis). Landmark analysis at 3 months. Continue blue line: no CA-AKI. Continue black line: CA-AKI. After the 3-month time point CA-AKI patients are stratified in those who recovered baseline renal function (dotted black line) vs. those who not recovered baseline renal function (pointed black line). CA-AKI: contrast associated acute kidney injury. 
Table 2. Univariate and multivariable Cox regression analysis for 3-month mortality.

\begin{tabular}{|c|c|c|c|c|c|c|}
\hline \multirow{2}{*}{--------------} & \multicolumn{3}{|c|}{ Univariate } & \multicolumn{3}{|c|}{ Multivariable } \\
\hline & HR & $95 \% \mathrm{CI}$ & $p$ & HR & $95 \% \mathrm{CI}$ & $p$ \\
\hline Age & 3 & 31 & - & 1.20 & 1.08 & 0.004 \\
\hline Female sex & 3.52 & $1.62-7.63$ & 0.001 & & & \\
\hline BMI & 1.01 & $0.91-1.35$ & $\overline{0.841}$ & & & \\
\hline Diabetes & 1.25 & $0.75-1.89$ & $\overline{0} . \overline{836}$ & & & \\
\hline Hypertension & $\overline{1} \cdot \overline{-}^{-}$ & $0.71-1.63$ & $\overline{0.740}$ & & & \\
\hline Hyperlipidemia & 0.89 & $0.65-1.62$ & 0.578 & & - & -- \\
\hline Current smoker & 1.04 & 0.73 & 0.827 & & & \\
\hline MI & 0.71 & $0.28-1.76$ & 0.713 & & & \\
\hline $\mathrm{PCI}$ & 0.77 & $0.53-1.10$ & 0.156 & & & \\
\hline CABG & 0.98 & $0.60-1.63$ & 0.950 & & & \\
\hline COPD & 1.07 & $0.25-4.48$ & 0.930 & & & \\
\hline PAD & 2.71 & $1.26-5.85$ & 0.011 & & & \\
\hline STEMI & 6.03 & $2.54-14.2$ & $<0.001$ & & & \\
\hline Killip class $\geq 2$ & $2 . \overline{4}-\overline{-}$ & $1.72-3.55$ & $<0.001$ & 2.21 & $1.08-2.84$ & 0.032 \\
\hline SBP & 0.99 & $0.97-1.03$ & 0.652 & & & \\
\hline Heart rate & 1.01 & $0.96-1.06$ & 0.783 & & & \\
\hline Hypotension & 1.82 & $0.76-4.54$ & 0.541 & & & \\
\hline Anemia & 1.96 & $1.44-2.68$ & $<0.001$ & 1.51 & $1.01-2.56$ & 0.045 \\
\hline IABP & 2.01 & $0.71-5.21$ & 0.653 & & & \\
\hline White blood cells & 1.07 & $1.02-1.12$ & 0.008 & & & \\
\hline Hemoglobin & 0.69 & $0.57-0.82$ & $<0.001$ & & & \\
\hline Albumin & $\overline{0 .} \overline{17}$ & $\overline{0.06-0.48}$ & $<0.001$ & & & \\
\hline Creatinine Clearance & 0.94 & $0.92-0.96$ & $<0.001$ & & & \\
\hline LVEF & 0.92 & $0.88-0.95$ & $<0.001$ & 0.89 & $0.83-0.95$ & $<0.001$ \\
\hline Troponin I at peak & 1.01 & $0.98-1.12$ & 0.496 & & & \\
\hline CK-MB at peak & 1.01 & $0.89-1.12$ & 0.189 & & & \\
\hline Radial access & 0.91 & $0.78-1.76$ & 0.654 & & & \\
\hline Multivessel disease & 1.80 & $1.12-2.50$ & 0.016 & & & \\
\hline Multivessel PCI & 0.86 & $0.62-1.22$ & 0.410 & & & \\
\hline ACE-inhibitor & $\overline{0.81}$ & $0.59-1.09$ & $\overline{0.14 \overline{8}}$ & & & \\
\hline $\begin{array}{c}\text { Angiotensin receptor } \\
\text { blocker }\end{array}$ & 0.56 & $0.38-1.19$ & 0.311 & & & \\
\hline$--\frac{-}{\text { Beta-blocker }}$ & $\overline{0.97}$ & $0.53-1.49$ & $\overline{0.442}$ & & & \\
\hline Statin & 0.91 & $0,64-1.78$ & 0.645 & & & \\
\hline Diuretic & 3.38 & $2.46-4.63$ & $<0.001$ & & & \\
\hline CA-AKI & 5.65 & $2.63-12.1$ & $<0.001$ & 3.51 & $1.05-7.01$ & 0.039 \\
\hline
\end{tabular}

HR: hazard risk. BMI: body mass index. MI: myocardial infarction. PCI: percutaneous coronary intervention. CABG: coronary artery bypass graft. COPD: chronic obstructive pulmonary disease. PAD: peripheral artery disease. STEMI: ST-segment elevation myocardial infarction. SBP: systolic blood pressure. IABP: intra-aortic balloon pump. LVEF: left ventricular ejection fraction. ACE: angiotensin converting enzyme. CA-AKI: contrast associated acute kidney injury.

\subsection{Three-Month Recovery of Renal Functioned in Patients with CA-AKI}

Out of 136 patients with CA-AKI, 123 reached the 3-month visit. Overall, 78 (63\%) patients showed partial or complete recovery of renal function to the baseline level within 3 months (recovered renal function group). Renal function remained impaired in 45 (37\%) patients (not recovered renal function group). Baseline characteristics of the two subgroups are shown in Table 1. Of note, 4 out of the 6 patients with severe CA-AKI and 3 out of 17 
with moderate CA-AKI in the index hospitalization did not recover baseline renal function. Prior CABG, peripheral artery disease, and lower baseline creatinine clearance were more common in patients with not recovered CA-AKI (Table 1). In the same group, MACD was lower and the need of contrast media overcame MACD in a higher percentage of cases (Table 1).

\subsection{CA-AKI and Mortality beyond 3-Month Visit}

Overall, 844 patients reached the 3-month time point. Afterwards, 134 (16\%) patients died. In particular, $96(13 \%)$ patients belonged to the no CA-AKI group, $17(22 \%)$ to the recovered renal function group and $21(47 \%)$ to the not recovered renal function group $(p<0.001)$. Unadjusted cumulative mortality beyond 3 months was higher in patients with not recovered renal function $(p=0.008$ vs. recovered renal function group and $p<0.001$ vs. no CA-AKI group) (Figure 1). After correction for potential confounding factors, the not recovered renal function group remained associated with an increased risk of mortality as compared to recovered renal function group and no CA-AKI subgroups (HR 2.01, 95\%CI 1.55-2.59, $p=0.009$ and HR 2.71,95\%CI 1.45-5.89, $p<0.001$, respectively). As compared to the no CA-AKI group, unadjusted cumulative mortality beyond the 3-month marker was higher in patients with recovered renal function $(p=0.043)$ (Figure 1). After correction for potential confounding factors, the difference was not significant (HR 1.07, 95\%CI 0.61-1.91, $p=0.751$ ) and patients of the recovered renal function group were not associated with a higher risk of mortality after the 3-month timepoint.

\subsection{Predictors of $C A-A K I$}

The analysis of predictors among baseline and procedural characteristics of CA-AKI was reported in Table 3. By multivariable analysis, baseline creatinine clearance, total amount of contrast media higher than the MACD, and Mehran risk score were the strongest predictors of CA-AKI (Table 3).

Table 3. Predictors of CA-AKI.

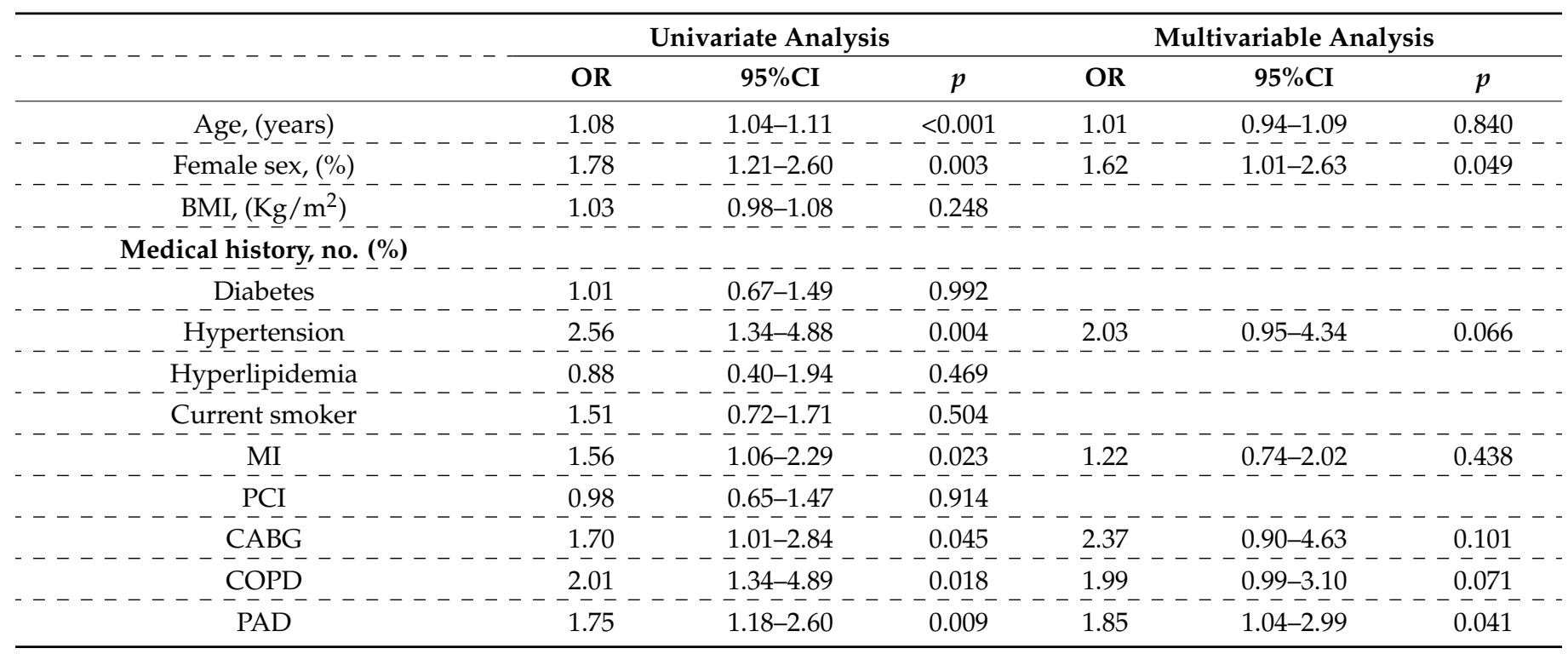


Table 3. Cont.

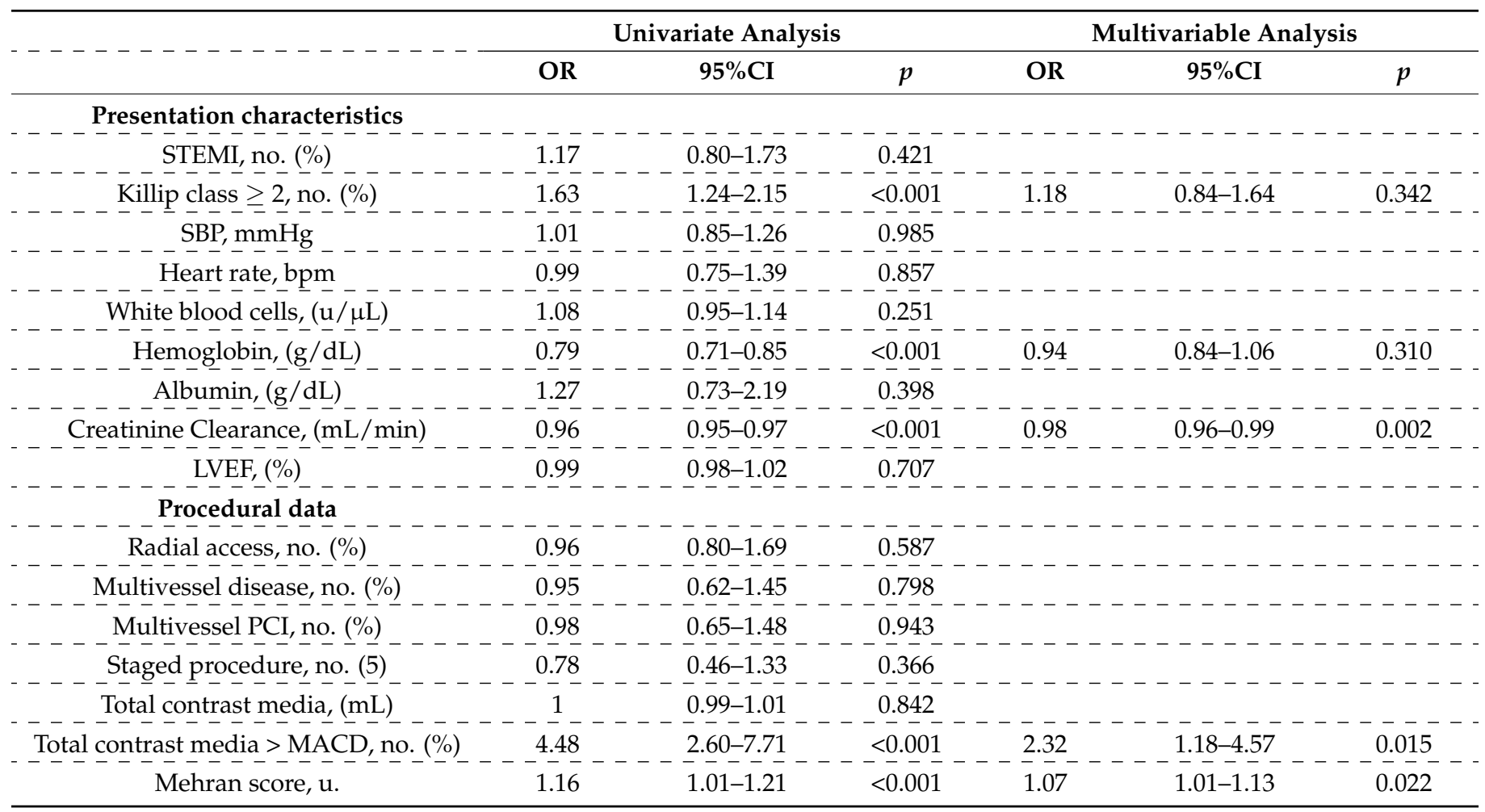

CA-AKI: contrast associated acute kidney injury. OR: odds ratio. BMI: body mass index. MI: myocardial infarction. PCI: percutaneous coronary intervention. CABG: coronary artery bypass graft. COPD: chronic obstructive pulmonary disease. PAD: peripheral artery disease. STEMI: ST-segment elevation myocardial infarction. SBP: systolic blood pressure. LVEF: left ventricular ejection fraction. MACD: maximum allowed contrast dose.

\section{Discussion}

The main findings of the present analysis can be summarized as follows:

- In older adults admitted to hospital for MI and undergoing invasive strategy with PCI, CA-AKI is a frequent complication occurring in $16 \%(95 \%$ CI $13-18 \%)$ of patients,

- baseline creatinine clearance, total amount of contrast media higher than the MACD and Mehran risk score emerged as independent predictors of CA-AKI,

- the occurrence of CA-AKI was independently associated with 3-month mortality,

- Among patients with CA-AKI, renal function did not return to baseline values after 3 months in 37\% (95\%CI 28-46\%), and these subjects showed a significantly higher risk of death.

The mean age of the population, and consequently the number of older adults admitted to hospital for MI, is increasing worldwide [16]. Older MI patients represents the subgroup at higher risk of death and early invasive strategy clearly showed its benefit in terms of the reduction of death and ischemic complications [16,18]. However, complications of invasive procedures tend to be frequent in older MI patients, and thus it is not surprising that advanced age is a well-established and strong risk factor for the occurrence of CA-AKI [10]. However, CA-AKI is a highly debated entity. Since the first reports in the 1950s [24], CA-AKI has been described both as a consequence of contrast media administration and as a silent, but deadly complication. In recent years, these assumptions have been challenged. The relationship between contrast administration and AKI is highly variable, strongly influenced by baseline risks, comorbidities, and other potential causes (i.e., atheromatous embolic debris) [25]. Similarly, some authors have suggested that CA-AKI is observed in subjects with several comorbidities, worse clinical presentation, complicated course of the disease, and then the increased mortality could be determined not by CA-AKI itself, but by the interaction of several factors of which CA-AKI is a bystander [11-13]. Our analysis is focused on older MI patients undergoing invasive strategy and PCI and 
represents an ideal subset of patients to try to clarify the missing pieces of the puzzle. Despite the inclusion of older MI patients, we did not find an excessively higher occurrence of CA-AKI as compared to previous studies on MI patients [26], and it was slightly lower as compared to that reported in the Comprehensive Evaluation of Risk Factors in Older Patients with AMI (SILVER-AMI) study (16\% vs. 19\%) [10].

We may suppose that these differences are explained by using modern contrast media and by the systematic application of hydration protocols. As in previous studies [8], we confirmed that patients experiencing CA-AKI have a worse short-term outcome. In addition, after correction for potential confounding factors, CA-AKI was an independent predictor of short-term death. This result further emphasizes the importance for physicians to minimize the occurrence of CA-AKI. As shown by previous trials [10], the first and most important step is the identification of patients at risk. The second step is the application of appropriate fluid administration. As suggested by current guidelines [27], hydration protocols were systematically applied, and we may hypothesize that they were able to reduce the burden of CA-AKI in our high-risk population. The third step is the minimization of contrast dose. Our analysis sheds new light on the importance of the MACD. We found that the relationship between contrast media and CA-AKI was not linear, but that contrast use is associated with CA-AKI when the MACD is exceeded. This finding deserves attention because it has several clinical implications. The total amount of contrast use is influenced by the experience of the operator and by the complexity and extension of the coronary anatomy requiring revascularization [25]. Complete revascularization should be considered the gold standard for MI patients [28]. However, evidence supporting this strategy has been mainly generated in patients with STEMI and a mean age of around 60 years [29]. In older patients with multivessel disease the potential benefit of a complete or extensive revascularization should be weighed against the risk of CA-AKI [30]. Operators should consider not to overcome MACD and eventually they should pursue a reasonable or physiology-guided coronary revascularization [30]. At the same time, it has to be noted that the risk profile of older MI patients with CA-AKI was significantly higher and more complex [26]. It is relevant to acknowledge that the design of the study (observational) is not enough to infer causality and to clearly demonstrate if CA-AKI is a direct or indirect determinant of death.

It has been reported that more than $50 \%$ of $\mathrm{MI}$ patients with CA-AKI have a complete or partial recovery of the renal function [25]. We confirmed this finding in our population of older MI patients. Around $60 \%$ of subjects with CA-AKI showed a return to baseline values of renal function after 3 months. Interestingly, this recovery is associated with an important clinical benefit, because beyond the 3-month time point, the rate of adverse events is similar to the one of patients without CA-AKI. On the contrary, the minority of patients without recovery of the renal function showed a significantly higher long-term mortality. However, our analysis did not allow for discriminating from the beginning who will have recovered or not recovered CA-AKI. Then, waiting for future studies able to clarify this issue, physicians can only pursue any effort to avoid CA-AKI.

\section{Study Limitations}

Our study has some limitations. First, this is a post hoc analysis and we cannot exclude the presence of unmeasured confounders. Second, the recruitment of patients was performed in a limited number of cardiology units. Therefore, the generalizability of our findings requires confirmation in a larger scenario. Third, we re-assessed renal function at a single time point ( 3 months after acute event) and only in patients suffering from CA-AKI in the index hospitalization. Fourth, in both studies (FRASER and HULK) the enrollment of the patients was performed at discharge. This has a huge implication in the interpretation of data because: (i) patients with low physical performance and high burden of comorbidities (including renal failure) have been excluded; (ii) patients transferred to another hospital or to another ward were not included in the analysis. Next, considering that current guidelines recommend hydrating patients undergoing coronary angiography [27], in our center all patients are treated with hydration before and after 
coronary angiography, and this might have reduced the risk of CI-AKI development in the study population.

\section{Conclusions}

Despite the use of modern contrast media and hydration protocols, CA-AKI occurs in a relevant portion of older MI patients undergoing invasive strategy and PCI. Patients with CA-AKI showed an increased risk of short-term death. Around two-thirds of the patients with CA-AKI recovered baseline renal function in the first 3 months, and their long-term prognosis was similar to that of patients without CA-AKI.

Author Contributions: Conceptualization, M.T., G.B., G.C. and S.B.; Data curation, R.P., E.T., R.C., E.M., G.F., A.F., G.M., M.S., F.V. and G.C.; Formal analysis, R.P., E.T., P.C., E.M., M.S., G.P. and F.V.; Funding acquisition, G.C.; Investigation, M.T., G.B., G.G., A.R., G.M., G.C. and S.B.; Methodology, G.B., P.C., E.M., G.G., G.F., A.F., A.R., G.C. and S.B.; Project administration, G.C. and S.B.; Software, G.P. and M.S.; Supervision, G.G.; Visualization, R.C. and S.B.; Writing-original draft, R.P., M.T., G.B., E.T., R.C., P.C., E.M., G.G., G.P., G.F., A.F., A.R., G.M., F.V., G.C. and S.B.; Writing-review \& editing, R.P., M.T., G.B., E.T., R.C., P.C., E.M., G.G., G.P., G.F., A.F., A.R., G.M., F.V., M.S., G.C. and S.B. All authors have read and agreed to the published version of the manuscript.

Funding: This work is partially supported by the grant of the Italian Health Ministry Ricerca Finalizzata 2018 (GR-2018-12367114).

Institutional Review Board Statement: The studies were conducted in accordance with the ethical principles of the Declaration of Helsinki. The ethical review boards of the participating hospitals approved both studies (for FRASER study approved by "Comitato Etico Unico della provincia di Ferrara" with ID number 140994; for HULK study approved by "Comitato Etico Unico della provincia di Ferrara" with ID number 161098). ClinicalTrials.gov, number NCT02386124 and NCT03021044.

Informed Consent Statement: All patients were informed that their participation was voluntary, and all of them gave written informed consent.

Data Availability Statement: The data presented in this study are available on request from the corresponding author.

Conflicts of Interest: The authors declare no conflict of interest.

\section{References}

1. Levy, E.M.; Viscoli, C.M.; Horwitz, R.I. The effect of acute renal failure on mortality: A cohort analysis. JAMA 1996, 275, 1489-1494. [CrossRef] [PubMed]

2. Solomon, R.J.; Mehran, R.; Natarajan, M.K.; Doucet, S.; Katholi, R.E.; Staniloae, C.S.; Sharma, S.K.; Labinaz, M.; Gelormini, J.L.; Barrett, B.J. Contrast-induced nephropathy and long-term adverse events: Cause and effect? Clin. J. Am. Soc. Nephrol. 2009, 4, 1162-1169. [CrossRef] [PubMed]

3. Brown, J.R.; Malenka, D.J.; DeVries, J.T.; Robb, J.F.; Jayne, J.E.; Friedman, B.J.; Hettleman, B.D.; Niles, N.W.; Kaplan, A.V.; Schoolwerth, A.C.; et al. Transient and persistent renal dysfunction are predictors of survival after percutaneous coronary intervention: Insights from the Dartmouth Dynamic Registry. Catheter. Cardiovasc. Interv. 2008, 72, 347-354. [CrossRef]

4. Goldenberg, I.; Chonchol, M.; Guetta, V. Reversible acute kidney injury following contrast exposure and the risk of long-term mortality. Am. J. Nephrol. 2009, 29, 136-144. [CrossRef] [PubMed]

5. Andreis, A.; Budano, C.; Levis, M.; Garrone, P.; Usmiani, T.; D’Ascenzo, F.; De Filippo, O.; D’Amico, M.; Bergamasco, L.; Biancone, L.; et al. Contrast-induced kidney injury: How does it affect long-term cardiac mortality? J. Cardiovasc. Med. (Hagerstown) 2017, 18, 908-915. [CrossRef] [PubMed]

6. Roghi, A.; Savonitto, S.; Cavallini, C.; Arraiz, G.; Angoli, L.; Castriota, F.; Bernardi, G.; Sansa, M.; De Servi, S.; Pitscheider, W.; et al. Impact of acute renal failure following percutaneous coronary intervention on long-term mortality. J. Cardiovasc. Med. (Hagerstown) 2008, 9, 375-381. [CrossRef] [PubMed]

7. Tsai, T.T.; Patel, U.D.; Chang, T.I.; Kennedy, K.F.; Masoudi, F.A.; Matheny, M.E.; Kosiborod, M.; Amin, A.P.; Messenger, J.C.; Rumsfeld, J.S.; et al. Contemporary incidence, predictors, and outcomes of acute kidney injury in patients undergoing percutaneous coronary interventions: Insights from the NCDR Cath-PCI registry. JACC Cardiovasc. Interv. 2014, 7, 1-9. [CrossRef]

8. Rihal, C.S.; Textor, S.C.; Grill, D.E.; Berger, P.B.; Ting, H.H.; Best, P.J.; Singh, M.; Bell, M.R.; Barsness, G.W.; Mathew, V.; et al. Incidence and prognostic importance of acute renal failure after percutaneous coronary intervention. Circulation 2002, 105, 2259-2264. [CrossRef] 
9. Madhavan, M.V.; Gersh, B.J.; Alexander, K.P.; Granger, C.B.; Stone, G.W. Coronary Artery Disease in Patients $\geq 80$ Years of Age. J. Am. Coll. Cardiol. 2018, 71, 2015-2040. [CrossRef]

10. Dodson, J.A.; Hajduk, A.; Curtis, J.; Geda, M.; Krumholz, H.M.; Song, X.; Tsang, S.; Blaum, C.; Miller, P.; Parikh, C.R.; et al. Acute Kidney Injury Among Older Patients Undergoing Coronary Angiography for Acute Myocardial Infarction: The SILVER-AMI Study. Am. J. Med. 2019, 132, e817-e826. [CrossRef]

11. Jacobs, F.M. Iodinated contrast and acute kidney injury: Major culprit or innocent bystander? Time for reappraisal. Crit. Care Med. 2013, 41, 1149. [CrossRef] [PubMed]

12. Lakhal, K.; Robert-Edan, V.; Ehrmann, S. In the Name of Contrast-Induced Acute Kidney Injury. Chest 2020, 157, 751-752. [CrossRef] [PubMed]

13. Dauerman, H.L.; Solomon, R.J. Innocent, Guilty, and Acute Kidney Injury. J. Am. Coll. Cardiol. 2020, 75, 1321-1323. [CrossRef] [PubMed]

14. Pavasini, R.; Maietti, E.; Tonet, E.; Bugani, G.; Tebaldi, M.; Biscaglia, S.; Cimaglia, P.; Serenelli, M.; Ruggiero, R.; Vitali, F.; et al. Bleeding Risk Scores and Scales of Frailty for the Prediction of Haemorrhagic Events in Older Adults with Acute Coronary Syndrome: Insights from the FRASER study. Cardiovasc. Drugs Ther. 2019, 33, 523-532. [CrossRef]

15. Campo, G.; Pavasini, R.; Maietti, E.; Tonet, E.; Cimaglia, P.; Scillitani, G.; Bugani, G.; Serenelli, M.; Zaraket, F.; Balla, C.; et al. The frailty in elderly patients receiving cardiac interventional procedures (FRASER) program: Rational and design of a multicenter prospective study. Aging Clin. Exp. Res. 2017, 29, 895-903. [CrossRef] [PubMed]

16. Campo, G.; Maietti, E.; Tonet, E.; Biscaglia, S.; Ariza-Solè, A.; Pavasini, R.; Tebaldi, M.; Cimaglia, P.; Bugani, G.; Serenelli, M.; et al. The Assessment of Scales of Frailty and Physical Performance Improves Prediction of Major Adverse Cardiac Events in Older Adults with Acute Coronary Syndrome. J. Gerontol. A. Biol. Sci. Med. Sci. 2020, 75, 1113-1119. [CrossRef]

17. Tonet, E.; Maietti, E.; Chiaranda, G.; Vitali, F.; Serenelli, M.; Bugani, G.; Mazzoni, G.; Ruggiero, R.; Myers, J.; Villani, G.Q.; et al. Physical activity intervention for elderly patients with reduced physical performance after acute coronary syndrome (HULK study): Rationale and design of a randomized clinical trial. BMC Cardiovasc. Disord. 2018, 18, 98. [CrossRef]

18. Campo, G.; Tonet, E.; Chiaranda, G.; Sella, G.; Maietti, E.; Mazzoni, G.; Biscaglia, S.; Pavasini, R.; Myers, J.; Grazzi, G. Exercise Intervention to Improve Functional Capacity in Older Adults After Acute Coronary Syndrome. J. Am. Coll. Cardiol. 2019, 74, 2948-2950. [CrossRef]

19. Campo, G.; Tonet, E.; Chiaranda, G.; Sella, G.; Maietti, E.; Bugani, G.; Vitali, F.; Serenelli, M.; Mazzoni, G.; Ruggiero, R.; et al. Exercise intervention improves quality of life in older adults after myocardial infarction: Randomised clinical trial. Heart 2020, 106, 1658-1664. [CrossRef]

20. Mehran, R.; Aymong, E.D.; Nikolsky, E.; Lasic, Z.; Iakovou, I.; Fahy, M.; Mintz, G.S.; Lansky, A.J.; Moses, J.W.; Stone, G.W.; et al. A simple risk score for prediction of contrast-induced nephropathy after percutaneous coronary intervention: Development and initial validation. J. Am. Coll. Cardiol. 2004, 44, 1393.e9.

21. Cigarroa, R.G.; Lange, R.A.; Williams, R.H.; Hillis, L.D. Dosing of contrast material to prevent contrast nephropathy in patients with renal disease. Am. J. Med. 1989, 86, 649-652. [CrossRef]

22. Chawla, L.S.; Bellomo, R.; Bihorac, A.; Goldstein, S.L.; Siew, E.D.; Bagshaw, S.M.; Bittleman, D.; Cruz, D.; Endre, Z.; Fitzgerald, R.L.; et al. Acute kidney disease and renal recovery: Consensus report of the Acute Disease Quality Initiative (ADQI) 16 Workgroup. Nat. Rev. Nephrol. 2017, 13, 241-257. [CrossRef] [PubMed]

23. Weisbord, S.D.; Palevsky, P.M.; Kaufman, J.S.; Wu, H.; Androsenko, M.; Ferguson, R.E.; Parikh, C.R.; Bhatt, D.L.; Gallagher, M.; PRESERVE Trial Investigators. Contrast-Associated Acute Kidney Injury and Serious Adverse Outcomes Following Angiography. J. Am. Coll. Cardiol. 2020, 75, 1311-1320. [CrossRef] [PubMed]

24. Bartels, E.D.; Brun, G.C.; Gammeltoft, A.; Gjorup, P. Acute anuria following intravenous pyelographyin a patient with myelomatosis. Acta MedScand. 1954, 150, 297-302.

25. McCullough, P.A.; Choi, J.P.; Feghali, G.A.; Schussler, J.M.; Stoler, R.M.; Vallabahn, R.C.; Mehta, A. Contrast-Induced Acute Kidney Injury. J. Am. Coll. Cardiol. 2016, 68, 1465-1473. [CrossRef]

26. Wi, J.; Ko, Y.G.; Kim, J.S.; Kim, B.K.; Choi, D.; Ha, J.W.; Hong, M.K.; Jang, Y. Impact of contrast-induced acute kidney injury with transient or persistent renal dysfunction on long-term outcomes of patients with acute myocardial infarction undergoing percutaneous coronary intervention. Heart 2011, 97, 1753-1757. [CrossRef] [PubMed]

27. Neumann, F.J.; Sousa-Uva, M.; Ahlsson, A.; Alfonso, F.; Banning, A.P.; Benedetto, U.; Byrne, R.A.; Collet, J.P.; Falk, V.; Head, S.J.; et al. 2018 ESC/EACTS Guidelines on myocardial revascularization. Eur. Heart J. 2019, 40, 87-165. [CrossRef]

28. Pavasini, R.; Biscaglia, S.; Barbato, E.; Tebaldi, M.; Dudek, D.; Escaned, J.; Casella, G.; Santarelli, A.; Guiducci, V.; Gutierrez-Ibanes, E.; et al. Complete revascularization reduces cardiovascular death in patients with ST-segment elevation myocardial infarction and multivessel disease: Systematic review and meta-analysis of randomized clinical trials. Eur. Heart J. 2020, 41, 4103-4110. [CrossRef]

29. Mehta, S.R.; Wood, D.A.; Storey, R.F.; Mehran, R.; Bainey, K.R.; Nguyen, H.; Meeks, B.; Di Pasquale, G.; López-Sendón, J.; Faxon, D.P.; et al. Complete Revascularization with Multivessel PCI for Myocardial Infarction. N. Engl. J. Med. 2019, 381, $1411-1421$. [CrossRef]

30. Biscaglia, S.; Guiducci, V.; Santarelli, A.; Amat Santos, I.; Fernandez-Aviles, F.; Lanzilotti, V.; Varbella, F.; Fileti, L.; Moreno, R.; Giannini, F.; et al. Physiology-guided revascularization versus optimal medical therapy of nonculprit lesions in elderly patients with myocardial infarction: Rationale and design of the FIRE trial. Am. Heart J. 2020, 229, 100-109. [CrossRef] 